Physics International 1 (1): 65-68, 2010

ISSN 1948-9803

(C) 2010 Science Publications

\title{
The Eclipsing X-Ray Binary System Centaurus X-3
}

\author{
Omar Al-Banawi \\ Department of Physics, Al-Hussein Bin Talal University, \\ P.O. Box 20, 71111, Ma'an, Jordan
}

\begin{abstract}
Problem statement: We present new physical and geometrical elements for the eclipsing X-ray binary system Krzemenski's star (Centaurus X-3). Approach: In this study, the procedure depended on getting the best fit between the observational photometric light curves and synthetic ones built up in accordance to the Roche model. Results: The estimated elements showed a good agreement with the early estimated ones. Conclusion: In building the synthetic light curves, we assumed that the optical component of the system is filling to Roche lobe, the system is rotationally synchronized and we neglecting the effect of the magnetic fields of both components.
\end{abstract}

Key words: Light curve, Centaurus X-3, X-ray binary stars, the physical and geometrical elements, compact object

\section{INTRODUCTION}

A binary stellar system is simply one in which two stars orbit around a common center of mass, that is they are gravitationally bound to each other. Almost 50 percent of the stars in the sky are part of a binary or multiple stellar systems.

A sub-class of binary systems are X-ray binaries, the systems in which a compact object (a white dwarf, neutron star or a black hole formed after collapse of an ordinary star) and a stellar companion, orbit each other at a distance small enough to enable mass transfer from the companion star to the compact object. The transferred matter spirals towards the compact object and forms an "accretion disk" around it.

The accreted gaseous matter is heated to very high temperatures $\left(10^{6}-10^{8} \mathrm{~K}\right)$, releasing the energy it acquired through gravitational infill in the form of $\mathrm{X}$ ray radiation.

In case of a neutron star or a white dwarf the emission originates from both the accretion disk and the surface of the compact star while in black hole binaries the only source of X-rays is the accretion disk. Figure 1 shows a sketch of an X-ray binary system as it would be seen by a nearby observer.

$\mathrm{X}$-ray binary stars are usually subdivided into two categories:

- High Mass X-ray Binaries (HMXB) where the companion's mass is greater than several solar masses, which enable it to eject matter through the stellar wind

- Low Mass X-ray Binaries (LMXB) where the companion's mass is around one solar mass and it provides matter through the inner Lagrangian point-the point where gravitational forces of the two stars and centrifugal force cancel

The first X-ray Source (Sco X-1) was discovered in 1962 during a rocket flight by a group led by Giacconi et al. (1964) Giacconi and Gursky (1974) who won the Nobel Prize for Physics in 2002 for his pioneering studies in X-ray astronomy.

The actual study of the X-ray binary stars began in the 1970s with the use of satellites equipped with $\mathrm{X}$-ray detectors. The first one, UHURU, was launched in 1970 (Giacconi et al., 1972). It mapped the X-ray sky in the energy range 2-6 keV. The Einstein observatory was launched in November 1978, It provided high-resolution images and accurate locations for thousands of X-ray sources (Harris et al., 1993).

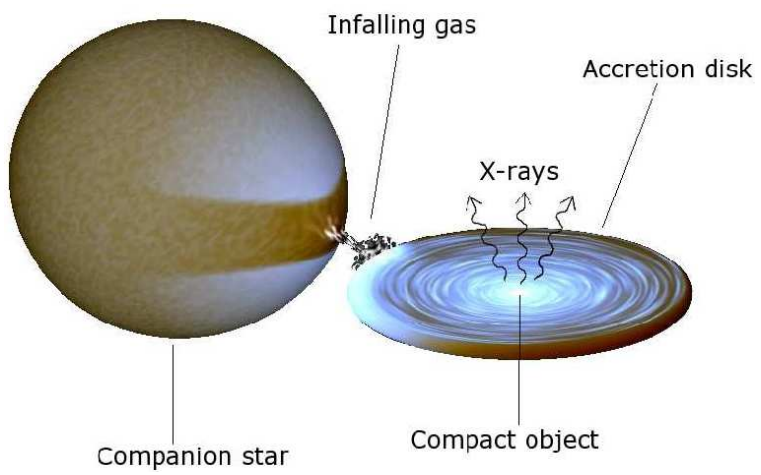

Fig. 1: A schematic view of an X-ray binary. Gas is pulled off the normal star by the gravity of the compact object 
Phy. Intl. 1 (1): 65-68, 2010

Table 1: The physical and geometrical elements of the system as given by earlier studies

\begin{tabular}{|c|c|c|}
\hline \multicolumn{2}{|l|}{ Parameter } & \multirow{2}{*}{$\begin{array}{l}\text { Reference } \\
\text { (Hutchings et al., 1979) } \\
\text { (Bahcall, 1978) }\end{array}$} \\
\hline Mass of the optical star & $\begin{array}{l}\mathrm{M}_{\mathrm{opt}}=(17 \pm 2) \mathrm{M}_{\alpha} \\
=(18.25 \pm 1.75) \mathrm{M}_{\alpha}\end{array}$ & \\
\hline Mass of the neutron star & $\begin{array}{l}\mathrm{M}_{\mathrm{n}}=(1.2 \pm 0.6) \mathrm{M}_{\mathrm{o}} \\
=(1 \pm 0.3) \mathrm{M}_{\mathrm{o}} \\
=(0.85 \pm 0.25) \mathrm{M}_{\mathrm{o}}\end{array}$ & $\begin{array}{l}\text { (Bahcall, 1978) } \\
\text { (Hutchings et al., 1979) } \\
\text { (Avni and Bahcall, 1975; Krzemenski, 1974) }\end{array}$ \\
\hline Eccentricity of the orbit & $\mathrm{e}<0.002$ & (Hutchings, 1974) \\
\hline Inclination of the system & $\mathrm{i}=90^{\circ}$ & (Giacconi and Ruffini, 1978) \\
\hline Orbital period & $\begin{array}{l}P_{\text {orb }}=2.087 d \\
=2.08712 d\end{array}$ & $\begin{array}{l}\text { (Schreier et al., 1972) } \\
\text { (Bagot, 1996) }\end{array}$ \\
\hline Orbital period change & $=-1.6 \mathrm{C} 10^{-6} \mathrm{sec}$ year $^{-1} \mathrm{P}_{\text {orb }}^{\bullet} / \mathrm{P}_{\text {orb }}$ & (Van den Heuvel, 1978) \\
\hline $\mathrm{X}$-ray luminosity & $\mathrm{L} \sim 7.7 \mathrm{C} 10^{37} \mathrm{erg} / \mathrm{s}$ & $\begin{array}{l}\text { (Bahcall, 1978) } \\
\text { (Schreier } \text { et al., 1972) }\end{array}$ \\
\hline Optical to X-ray luminosity ratio & $\mathrm{L}_{\mathrm{opt}} / \mathrm{L}_{\mathrm{x}}=100$ & (Bahcall, 1978) \\
\hline Pulsation period & $\mathrm{P}_{\mathrm{p}}=4.84 \mathrm{~s}$ & (Bahcall, 1978) \\
\hline Distance from earth & $5-10 \mathrm{kpc}$ & (Krzemenski, 1974) \\
\hline Visual magnitude & $\mathrm{m}_{\mathrm{v}}=13.4 \mathrm{mag}$ & (Hutchings, 1974) \\
\hline & $\Delta \mathrm{m}=0.7 \mathrm{mag}$ & (Avni, 1978b) \\
\hline & $\Delta \mathrm{m}=0.8 \mathrm{mag}$ & (Al-Wardat et al., 1998) \\
\hline Duration of X-ray eclipse & $\mathrm{Je}=39 \pm 2^{\circ}$ & (Al-Wardat et al., 1998) \\
\hline Mass ratio of the system & $q=0.06 \pm 0.002$ & (Al-Wardat et al., 1998) \\
\hline Radius of the optical component & $r=0.625 \pm 0.003$ & (Al-Wardat et al., 1998) \\
\hline Limb darkening coefficient & $\mathrm{u}=0.8 \pm 0.02$ & (Al-Wardat et al., 1998) \\
\hline Gravity darkening coefficient & $\mathrm{t}_{\mathrm{o}}=0.4 \pm 0.02$ & (Al-Wardat et al., 1998) \\
\hline Efficiency of absorption and re-emission & $\eta=0.1 \pm 0.05$ & (Al-Wardat et al., 1998) \\
\hline Optical to X-ray luminosity ratio & $\mathrm{L}_{\mathrm{opt}} / \mathrm{L}_{\mathrm{x}}=100 \pm 5$ & (Al-Wardat et al., 1998) \\
\hline Effective temperature of the optical star & $\mathrm{T}_{\text {eff }}=38000 \mathrm{~K} \pm 1000$ & \\
\hline
\end{tabular}

The European X-Ray Observatory Satellite (EXOSAT) operated from May 1983 to April 1986 allowed to perform continuous observations of X-ray sources lasting several days without the interference of Earth occultation's. These and later missions (Chandra, XMM-Newton, Rossi XTE, BeppoSAX, INTEGRAL and others) observed X-ray radiation from different sources.

As a result of this development by a number of scientists to the set of results shown in Table 1, which represents the previous studies of this system Centaurus $\mathrm{X}-3$.

Ground-based observations with optical telescopes have demonstrated that X-ray sources are often members of binary systems. A fraction of these sources are eclipsing systems that show periodic light variations with time, known as the light curves.

Many contributions have been made to study and analyze the light curves of the X-ray eclipsing binaries, such as (Hutchings, 1978; Avni, 1978a; Antokhina and Cherpashchuk, 1987; 1994; Al-Wardat et al., 1998; Sabat et al., 1998) and others. The present study focuses on the analysis of one such binary, Centaurus $\mathrm{X}-3$, in a step to derive more reliable physical and geometrical elements.

Specifications of the system Centaurus X-3 (Krzemenski's star): The eclipsing X-ray binary system Krzemenski's star (Centaurus X-3) is known as a high-mass X-ray binary system consists of $18 \mathrm{M}_{\odot}$ O6.5V-III giant optical star and a $1.2 \mathrm{M}_{\odot}$ neutron star (Bahcall, 1978).

The X-ray star Cen X-3 was discovered from the UHURU observation by Giacconi et al. (1971) where it has been distinguished as 4.84 Second X-ray pulsar with a 2.087 day eclipse period. Krzemenski (1974) declared that the X-ray star Cen X-3 is a component of an eclipsing binary system consists of the star Cen X-3 and a giant optical star of the spectral type B0, with optical magnitude $13.4 \mathrm{mag}$ at a distance of $10 \mathrm{kpc}$.

There are other issued values of the spectral type of the star, these are:

- $\quad$ O6.5II-Ve (Van den Heuvel, 1981)

- O6.5V-III (Hoffmeister et al., 1985)

- O6f (Hutchings, 1978)

The light curve of this system characterized by two maximums at phases (0.25 and 0.75) because the observer faces the maximum area of the system and two minimums at phases $(0.0$ and 0.5$)$, one results from the eclipse of the compact object to the optical star and the other the deeper results from the cold hemisphere of the optical star. 


\section{MATERIALS AND METHODS}

Our method of determining the elements of eclipsing X-ray binaries depends on iterated attempts of getting the best fitting between the optical observational light curve and a synthetic one. The standard model of X-ray binaries was used in synthesizing light-curves of the system. The synthetic light curves were built in accordance to Roche model and taking into account the X-ray reflection and heating of the opposite side of the optical companion. We followed Sabat et al. (1998) in their solution to the Roche Model's basic equation, where they used Newton-Raphson method to solve it, after changing it into an eighth-degree polynomial equation, which proved to be more precise and yet, more efficient than the series solutions developed by Kopal (1959).

We considered the input elements of the best fit as the elements of the system. These elements are; mass ratio of the system q, fractional radius of the optical component $\mathrm{r}$, limb darkening coefficient $\mathrm{u}$, gravity darkening coefficient $\tau_{0}$, efficiency of absorption and re-emission $\eta$, optical $X$-ray luminosity ratio $L_{\text {opt }} / L_{x}$ and the effective temperature of the optical star $\mathrm{T}_{\text {eff. }}$.

Table 2: New elements of Centaurus X-3

\begin{tabular}{ll}
\hline Mass ratio of the system & $\mathrm{q}=0.062 \pm 0.001$ \\
\hline Radius of the optical component & $\mathrm{r}=0.63 \pm 0.002$ \\
Limb darkening coefficient & $\mathrm{u}=0.82 \pm 0.02$ \\
Gravity darkening coefficient & $\tau=0.42 \pm 0.01$ \\
Efficiency of absorption and re-emission & $\eta=0.12 \pm 0.04$ \\
Optical to X-ray luminosity ratio & $\mathrm{L}_{\mathrm{op}} / \mathrm{L}_{\mathrm{x}}=103 \pm 4$ \\
\hline
\end{tabular}

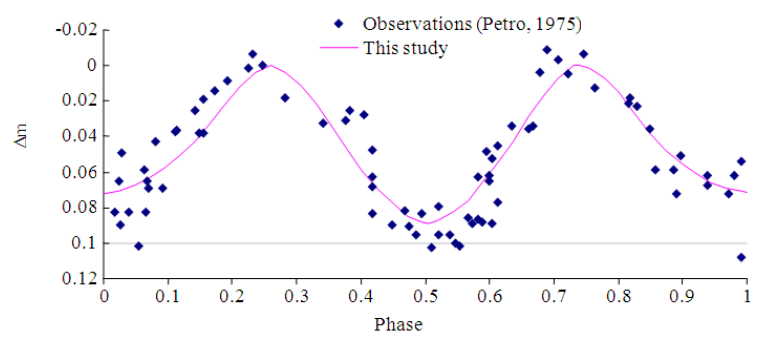

Fig. 2: The estimated synthetic light curve against the observations of Petro (1975)

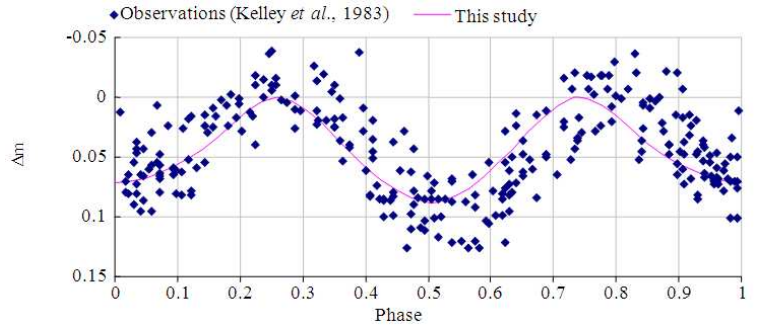

Fig. 3: The estimated synthetic light curve against the observations of Kelley et al. (1983)
Many attempts were performed to get the best fit. As an observational reference and guide, we used the observational light curves of Petro (1975) and (Kelley et al., 1983).

\section{RESULTS}

The best fit was achieved using a synthetic light curve represented by the new physical and geometrical elements listed in Table 2.

The achieved best between different observation light curve and our synthetic one are represented by Fig. 2 and 3. Where Fig. 2 shows our synthetic light curve against the observations of Petro (1975), Fig. 3 shows it against the observations of Kelley et al. (1983).

\section{DISCUSSION}

The importance of this research required obtaining the values of elements of physical and geometrical required to verify compliance with other observational as well as it gives a clearer image and explains this dual system Centaurus X-3.

\section{CONCLUSION}

New physical and geometrical elements of the Xray eclipsing binary system Centaurus X-3 (Krzemenski's star) were found. The procedure depended on getting the best fit between the observational photometric light curves, which measured using ground based telescopes and a synthetic light curve built up in accordance to the Roche model.

In building the synthetic light curves, we assumed that the optical component of the system is filling to Roche lobe, the system is rotationally synchronized and we neglecting the effect of the magnetic fields of both components.

\section{REFERENCES}

Al-Wardat, M.A.S., H.M. Al-Naimiy, I.A. Barghouthi and H.A. Sabat, 1998. New elements for three eclipsing X-Ray binary systems: HZ Herculis, Centaurus X-3 and VELA X-1. Astrophys. Space Sci., 260: 335-345. 10.1023/A: 1001714812242

Antokhina, E.A. and A.M. Cherepashchuk, 1994. Synthesizing of radial velocity curves of close binary systems. Astronomicheskij Zhurnal, 71: 420-430.

http://adsabs.harvard.edu/abs/1994AZh....71..420A 
Antokhina, E.A. and A.M. Cherpashchuk, 1987. SS 433-the parameters of the eclipsing system with a processing thick accretion disk. Astronomicheskii Zhurnal, 64: 562-585.

Avni, Y. and J.N. Bahcall, 1975. Ellipsoidal light variations and masses of X-ray binaries. Astrophys. J., 197: 675-688. DOI: 10.1086/153558

Avni, Y., 1978a. Mass Estimates from Optical Light Curves for Binary X-Ray Sources. In: Physics and Astrophysics of Neutron Stars and Black Holes, Giacconi, R. and R. Ruffini (Eds.). North-Holland Publishing Company, Amsterdam, pp: 43-62.

Avni, Y., 1978b. On the contribution of active galactic nuclei to the diffuse X-ray background. Astron. Astrophys., 63:

13-16. http://articles.adsabs.harvard.edu//full/1978A\%26 A...63L..13A/L000013.000.html

Bagot, P., 1996. Boost of the orbital motion in high mass X-ray binaries. Astron. Astrophys., 314: 576-584. http://adsabs.harvard.edu/full/1996A\%26A...314..5 $76 \mathrm{~B}$

Bahcall, J.N., 1978.Optical Properties of Binary X-Ray Sources. In: Physics and Astrophysics of Neutron Stars and Black Holes, Giacconi, R. and R. Ruffini (Eds.). North-Holland Publishing Company, Amsterdam, pp: 63-110.

Giacconi, R. and H. Gursky, 1974. X-Ray Astronomy. 1st Edn., Springer, Holland, ISBN: 9027702950, pp: 460 .

Giacconi, R. and R. Ruffini, 1978. Physics and Astrophysics of Neutron Stars and Black Holes. 1st Edn., North-Holland Pub. Co., New York, pp: 866.

Giacconi, R., H. Gursky, J.R. Waters, G. Clark and B. Rossi, 1964. Two sources of cosmic X-rays in Scorpius and Sagittarius. Nature, 204: 981-982. DOI: $10.1038 / 204981 \mathrm{a} 0$

Giacconi, R., H. Gursky and E. Kellogg, E. Schreier and H. Tananbaum. 1971. Discovery of periodic X-ray pulsations in centaurus X-3 from HURU. Astrophys. J., 167: 67-73. DOI: 10.1086/180762

Giacconi, R., S. Murray, H. Gursky, E. Kellogg and E. Schreier et al., 1972. The Uhuru catalog of X-ray sources. Astrophy. J., 178: 281-308. http://adsabs.harvard.edu/full/1972ApJ...178..281G

Harris, M.J., G.H. Share, M.D. Leising and J.E. Grove, 1993. SMM observations of gamma-ray transients. I. A search for variable emission at $\mathrm{MeV}$ energies from five galactic and extragalactic sources. Astrophys. J., 416: 601-619. DOI: 10.1086/173262
Hoffmeister, C., G. Richter and W. Wenzel, 1985. Variable Stars. 1st Edn., Springer-Verlag, Berlin, ISBN: 3540134034, pp: 328.

Hutchings, J.B, A.P. Cowely, D. Crampton, J. van Paradijs and N.E. White, 1979. Centaurus X-3. Astrophys. J., 229: 1079-1084. DOI: 10.1086/157042

Hutchings, J.B., 1974. The synthesis of close-binary light curves. VI. X-ray and collapse binaries. Astrophys. J., 188: 341-348. DOI: 10.1086/152722

Hutchings, J.B., 1978. Optical Observations of X-Ray Sources. In: Physics and Astrophysics of Neutron Stars and Black Hole. Giacconi, R. and R. Ruffini (Eds.). North Holland Publishing Co., Amsterdam, pp: 202-215.

Kelley, R.L., S. Rappaport, G.W. Clark and L.D. Petro, 1983. Orbital period changes in Centaurus X-3. Astrophysical J., 268: 790-799. DOI: $10.1086 / 161001$

Kopal, Z., 1959. Close Binary Systems. 1st Edn., Wiley, New York, ISBN: 0471503258, pp: 558.

Krzemenski, W., 1974. The identification and UBV photometry of the visible component of the Centaurus X-3 binary system. Astrophys. J., 192: L135-L138. DOI: 10.1086/181609

Petro, L.D., 1975. Photoelectric observations of Krzeminski's star, the companion of Centaurus X3. Astrophys. J., 195: 709-713. DOI: $10.1086 / 153372$

Sabat, H.A., H.M. Al-Naimiy, I.A. Barghouthi and M.A. Al-Wardat, 1998. Synthetic light-curves of some eclipsing X-ray binary systems. Astrophys. Space Sci., 260: 347-357. DOI: 10.1023/A:1001766829080

Schreier, E., R. Levinson, H. Gursky, E. Kellogg and H. Tananbaum et al., 1972. Evidence for the Binary nature of Centaurus X-3 from UHURU Xray observations. Astrophys. J., 172: L79-L89. DOI: $10.1086 / 180896$

Van den Heuvel, E.P.J., 1978. Evolution of Close Binaries, with Possible Applications to Massive XRay Binaries. In: Physics and Astrophysics of Neutron Stars and Black Holes, Giacconi, R. and R. Ruffini (Eds.). North Holland Publishing Co., Amsterdam, pp: 828-871.

Van den Heuvel, E.P.J., 1981. X-Ray Binaries and Stellar Evolution. In: X-Ray Astronomy, Space Science Reviews, Anderson, R.D. (Ed.). D Reidel Publishing Company, Dordrecht, pp: 309-309. 